\title{
Reference ranges for the linear dimensions of the intracranial ventricles in preterm neonates
}

\author{
M W Davies, M Swaminathan, S L Chuang, F R Betheras
}

\begin{abstract}
Aims-To establish normal ranges, in preterm infants < 33 weeks' gestation, for measurements of the lateral, third, and fourth ventricles and to assess intraobserver and inter-observer reliability. To assess the effect of head position during scanning on lateral ventricle size. To determine whether sex influences ventricle size.

Methods-A prospective study involving infants < 33 weeks' gestational age (GA) at birth. Cranial ultrasound scans were done during the first 3 days of life. Linear dimensions of the anterior horn width and thalamo-occipital distance of the lateral ventricles, the width of the third ventricle, and the width and length of the fourth ventricle were measured. Measurements were plotted against $G A$ and reference ranges produced. All measurements were tested for intra-observer and interobserver reliability. Head position and sex differences were studied.
\end{abstract}

Results-120 infants with known GA $\left(23^{+1}\right.$ to $32^{+6}$ weeks) had their intracranial ventricles measured. Reference ranges obtained were-anterior horn width: 0-2.9 $\mathrm{mm}$; thalamo-occipital distance: 8.7-24.7 $\mathrm{mm}$; third ventricle width: 0-2.6 mm; fourth ventricle width: $3.3-7.4$ $\mathrm{mm}$; fourth ventricle length: $2.6-6.9 \mathrm{~mm}$. Dependent and non-dependent lateral ventricles did not differ significantly in size. There was no clinically significant difference in ventricular size between sexes.

Conclusions-Reference ranges for the measurement of the intracranial ventricles in preterm infants from 23 to 33 weeks' GA are provided and can be used in the diagnosis and assessment of ventricular enlargement in preterm infants. All measurements have good intra-observer and inter-observer reliability. Head position at the time of scanning does not influence the asymmetry of the lateral ventricular measurements. The infant's sex does not influence ventricular size.

(Arch Dis Child Fetal Neonatal Ed 2000;82:F218-F223)

Keywords: preterm infants; cranial ultrasound; ventricular measurement; reference range

Ultrasonography is a well established, noninvasive diagnostic tool used in neonatal intensive care units to measure intracranial ventricular size. ${ }^{1}$ Quantitative measures of ventricular size are superior to any subjective assessment. ${ }^{2}$
Numerous methods to measure ventricular size in newborns have been published. ${ }^{3-11}$ Data on the dimensions of the anterior horn and body of the lateral ventricles are widely available for preterm newborns. However, there are no reference data available on the linear dimensions of the occipital horns of the lateral ventricle or the third and fourth ventricles in preterm infants. ${ }^{1}$ In addition, intra-observer and interobserver reliability have not been well investigated for any ventricular measurement. The aims of our study were to: (1) establish normal value ranges, in preterm infants less than 33 weeks' gestation, for measurements of the lateral, third, and fourth ventricles; (2) assess intra-observer and inter-observer reliability for all ventricular measurements; (3) assess the effect of head position during the scan on lateral ventricle size; and (4) determine whether sex influences ventricle size.

\section{Methods}

This was a prospective study involving infants born from 8 December 1997 to 28 August 1998. In our unit, routine cranial ultrasound scans are done on all infants $<1500 \mathrm{~g}$ birthweight and/or less than 33 weeks' gestational age on days 1, 3, 7, and 28. All infants less than 33 weeks were eligible for our study. Infants were excluded if the gestational age was not known, if there was any craniospinal malformation, or if there was grade 3 or 4 intraventricular haemorrhage according to the classification of Papile et al. ${ }^{12}$ Measurements were taken from the first cranial scan done within the first 3 days of life. The gestational age assessment was based upon an early pregnancy scan (< 18 weeks' gestational age) or by certain dates (date of last menstrual period) if no antenatal scan was performed.

Cranial ultrasound scans were done with a General Electric LOGIQ 500 scanner (GE Medical Systems, Waukesha, USA) with a $7 \mathrm{MHz}$ curvilinear sector probe (GE LOGIQ; C721). The scans were done by one of the four authors and each scan was subsequently validated by the senior sonologist (FRB). The infant's head was not moved from its original resting position when first approached to be scanned. The resting position of the infant's head was noted as either right side up, left side up, or face up. The methods for measuring each intraventricular dimension are described below. All ventricular measurements were taken with calipers placed at the junction of the ependymal lining and ventricular cavity. Figure 1 shows the caliper position and alignment for all measurements. 


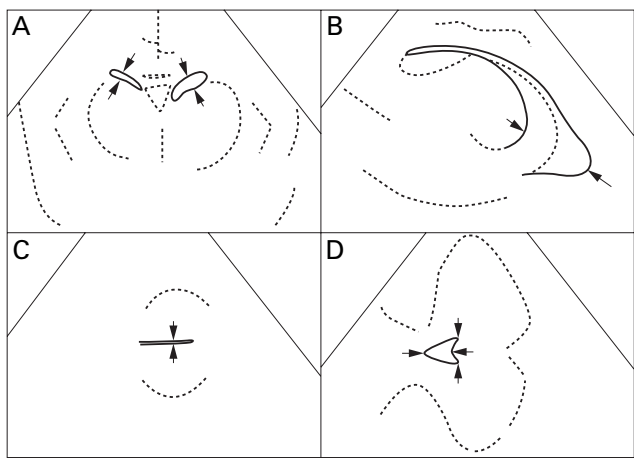

Figure 1 A line drawing showing the measurements made on the ultrasound images in figs 2-5 (A-D, respectively), with arrows indicating the correct caliper position and alignment.

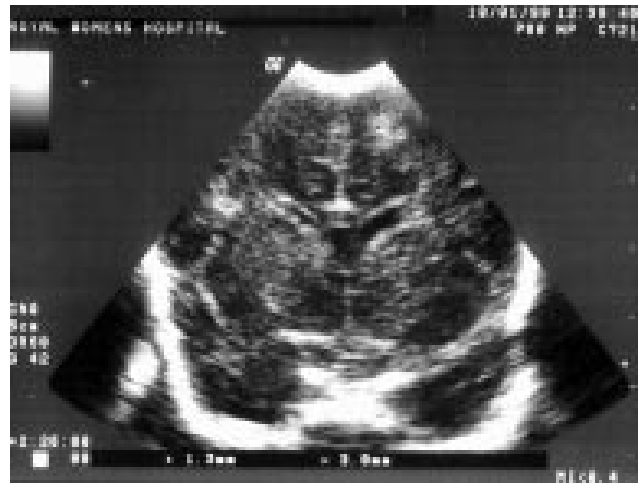

Figure 2 A coronal section of the brain on ultrasound showing measurement of the anterior horn width of the lateral ventricles.

THE ANTERIOR HORN WIDTH

The width of the anterior horn of the lateral ventricles was measured in a coronal view, with the plane of the scan at the level of the interventricular foramina of Monro (just anterior to the choroid plexus in the third ventricle). The width was measured on each side as the distance between the medial wall and floor of the lateral ventricle at the widest point (figs $1 \mathrm{~A}$ and 2$).{ }^{513}$

THE THALAMO-OCCIPITAL DISTANCE

This measurement was taken in the oblique parasagittal view, which demonstrated the entire lateral ventricle with the anterior horn, the atrium, and the temporal and occipital horns (to its maximal extent) all in view. The thalamo-occipital distance was then measured from the outermost point of the thalamus, at its junction with the choroid plexus, to the outermost part of the occipital horn posteriorly (figs $1 \mathrm{~B}$ and 3$)$.

THE THIRD VENTRICLE WIDTH

The measurement of the third ventricle was taken in the axial plane between the thalami and in line with the foramina of Monro (at the level just above a line from the outer canthus of the eye to the upper point of insertion of the ear). The measurement was taken from inner edge to inner edge (figs $1 \mathrm{C}$ and 4 ).

THE FOURTH VENTRICLE WIDTH AND LENGTH The fourth ventricular width and length were measured in an oblique/transverse plane through the posterior fossa using the asterion

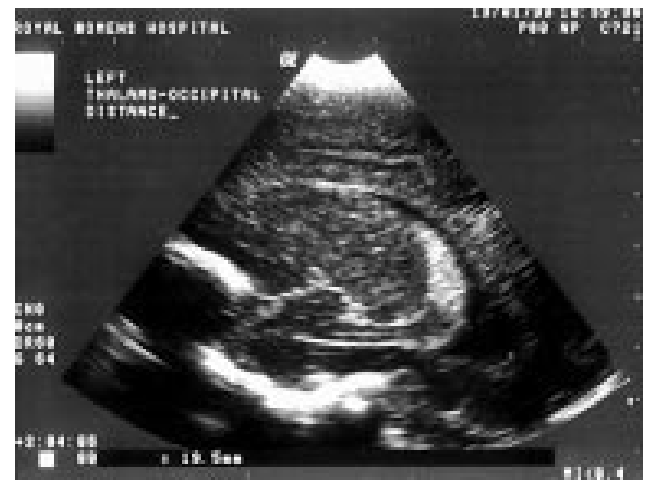

Figure 3 A parasaggital section of the brain on ultrasound showing measurement of the thalamo-occipital distance of the lateral ventricle.

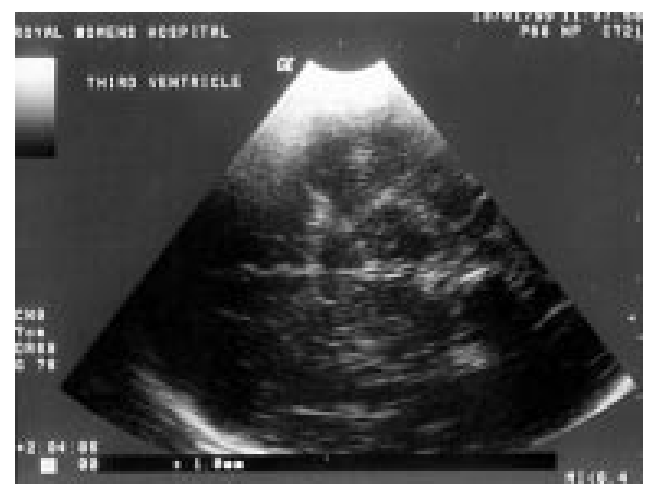

Figure 4 A transverse section of the brain on ultrasound showing measurement of the width of the third ventricle.

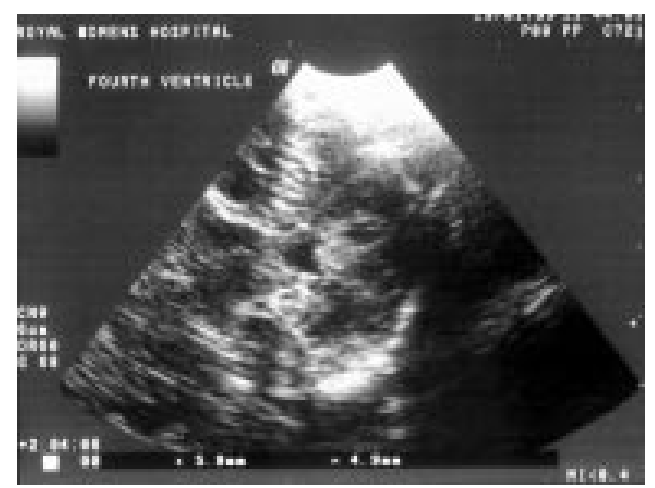

Figure 5 An oblique/transverse section of the cerebellum on ultrasound showing measurement of the width and length of the fourth ventricle.

as the acoustic window. The asterion is the junction of the parieto-occipital and lambdoid sutures and is palpable just behind the pinna of the ear. This plane is parallel and posterior to the longitudinal plane of the brainstem, producing a triangular view of the fourth ventricle through the lateral recesses. The width of the fourth ventricle is the distance between the lateral recesses and forms the base of the triangle, posterior to which is the cerebellar vermis. The length of the fourth ventricle is the distance from the base to the apex, which is the end of the cerebral aqueduct. The lateral walls are formed by the cerebellar peduncles (figs 1D and 5). 
Table 1 Mean (SD) ventricular measurements according to sex

\begin{tabular}{llcl}
\hline Measurement (mm) & Girls & Boys & p Value \\
\hline Anterior horn width & $1.22(0.85)$ & $1.30(0.79)$ & 0.44 \\
Thalamo-occipital distance & $15.6(3.4)$ & $17.5(4.3)$ & 0.0002 \\
Third ventricle width & $0.99(0.36)$ & $1.12(0.93)$ & 0.3 \\
Fourth ventricle width & $5.30(1.07)$ & $5.42(1.00)$ & 0.56 \\
Fourth ventricle width & $4.73(1.15)$ & $4.75(1.06)$ & 0.92 \\
\hline
\end{tabular}

Table 2 Difference between two observers for intracranial ventricular measurements

\begin{tabular}{lclll}
\hline Measurement $(\mathrm{mm})$ & $\begin{array}{l}\text { Mean (SD) of average } \\
\text { between measurements }\end{array}$ & $\begin{array}{l}\text { Mean } \\
\text { difference }\end{array}$ & Variability & $\begin{array}{l}\text { Intraclass } \\
\text { correlation } \\
\text { coefficient }\end{array}$ \\
\hline Right AHW & $1.01(0.57)$ & 0.01 & 0.28 & 0.89 \\
Left AHW & $1.29(0.83)$ & 0.02 & 0.32 & 0.93 \\
Right TOD & $16.6(3.8)$ & 0.06 & 1.92 & 0.88 \\
Left TOD & $17.6(2.9)$ & 0.23 & 1.94 & 0.80 \\
Third ventricle width & $0.77(0.29)$ & 0.01 & 0.20 & 0.79 \\
Fourth ventricle width & $5.40(0.73)$ & 0.15 & 0.70 & 0.63 \\
Fourth ventricle length & $5.55(0.90)$ & 0.02 & 1.22 & 0.38 \\
\hline
\end{tabular}

The average between measurements refers to the average of the mean of both repeated measurements.

The mean difference refers to the average difference of the repeated measurements.

Variability refers to the standard deviation of the differences between repeated measurements.

$\mathrm{AHW}$, anterior horn width; TOD, thalamo-occipital distance.

INTRA-OBSERVER AND INTER-OBSERVER

RELIABILITY

To assess intra-observer reliability the first 30 measurements for each ventricle (done on infants in our study population) were repeated by the same observer (MWD or MS). Usually, the optimal two dimensional image is frozen on the ultrasound screen and the measurement taken. For the repeat measurement the image was unfrozen, the ultrasound probe reapplied to the infants head, the optimal image obtained again, and another measurement taken.

Inter-observer reliability was assessed in a separate population of infants (all < 33 weeks' gestational age) not included in our study population. Thirty infants had measurements performed by one observer and the measurements were then repeated by the second observer who was blinded to the first observer's data. These measurements were done by MWD and MS.

The average difference between measurements should be close to zero and the variability between measurements is defined as the standard deviation of the differences between repeated measurements. The intraclass correlation coefficient as described by Jamart ${ }^{14}$ was calculated for each variable and the strength of agreement scale of Brennan and Silman ${ }^{15}$ was used for interpretation. The strength of agreement or reliability was poor if the intraclass correlation coefficient was less than 0.20 , fair if between 0.21 and 0.40 , moderate if between 0.41 and 0.60 , good if between 0.61 and 0.80 , and very good if $\geqslant 0.81$.

\section{Results}

There were 225 infants less than 33 weeks' gestational age admitted to our unit during the study period. One hundred and twenty infants with a known gestational age (ranging from $23^{+1}$ to $32^{+6}$ weeks) had their intracranial ventricles measured. The mean (SD) birthweight was 1222 (405) g and the mean (SD) gestational age was 29.2 (2.5) weeks. The gestational age assessment was based upon an early pregnancy scan ( $<18$ weeks' gestational age) in $108(90 \%)$ cases or by certain dates (date of last menstrual period) in $12(10 \%)$ cases if no antenatal scan was performed. One hundred and five babies were excluded because of: uncertain gestation (14), congenital malformation (three), or grade 3 or 4 intraventricular haemorrhage (four). A further 84 infants did not have a scan done by day 3 .

All but two babies had unequal right and left thalamo-occipital distance measurements. The median difference was $2.3 \mathrm{~mm}$ (interquartile range, 1.2 to 3.8 ). There were 18 infants with a difference greater than $5 \mathrm{~mm}$. At the time of the scan, 54 infants were lying with their heads on the side with the right side up, 61 with the left side up (three were face up and two unrecorded). The mean (SD) anterior horn width of the non-dependent ventricle was 1.3 $(0.8) \mathrm{mm}$ and for the dependent ventricle it was $1.2(0.8) \mathrm{mm}(\mathrm{p}=0.3)$. The mean $(\mathrm{SD})$ thalamo-occipital distance of the nondependent ventricle was 16.7 (4.2) $\mathrm{mm}$ and for the dependent ventricle it was 16.6 (3.9) $\mathrm{mm}$ $(\mathrm{p}=0.89)$. There was no effect of head position on size of the lateral ventricles. Therefore, data from both sides were pooled for all further analysis.

Table 1 displays the ventricular dimensions according to sex. The only significant difference between the sexes was the mean thalamooccipital distance, which was $1.9 \mathrm{~mm}$ longer in boys. Because this difference is not clinically significant, it is similar to the variability between observers (table 2), and there were no other differences between the sexes, the data were combined for all further analysis.

Figures 6 to 10 show the scatter plots for the ventricular measurements according to gestational age. Regression equations and coefficients of determination $\left(R^{2}\right)$ are also shown. All measurements show little or no change with gestational age. The greatest degree of change with gestational age is seen in the fourth ventricle width, with an $R^{2}$ of 0.124 , indicating that only $12 \%$ of this measurement is accounted for by gestational age. All the other measurements have much lower values for $R^{2}$. Because of the lack of change in ventricular measurements with gestational age (from 23 to $32+6$ weeks), measurements across these gestational age can be combined. Pooled data from both lateral ventricles, both sexes, and all gestational ages gives summary statistics with mean and standard deviation, with appropriate reference ranges, for all ventricular measurements (table 3).

Tables 2 and 4 show the results of reliability testing. All mean differences are close to zero. The intra-observer agreement is very good for all measurements except the fourth ventricle length, which is still good. The inter-observer reliability is very good for anterior horn width and right thalamo-occipital distance, good for left thalamo-occipital distance, third ventricle width, and fourth ventricle width, but only moderate for fourth ventricle length. 


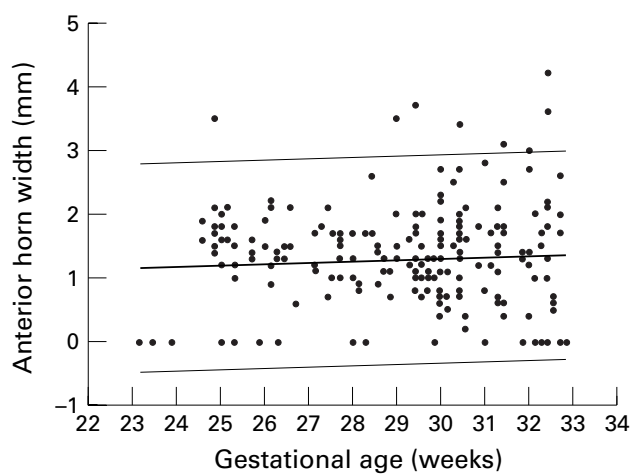

Figure 6 Anterior horn width $(A H W)$ by gestational age (GA) with fitted line plot (bold line) and $95 \%$ confidence intervals (thin lines). $A H W(\mathrm{~mm})=0.71+0.019 \times G A$ (weeks); $R^{2}=0.004$

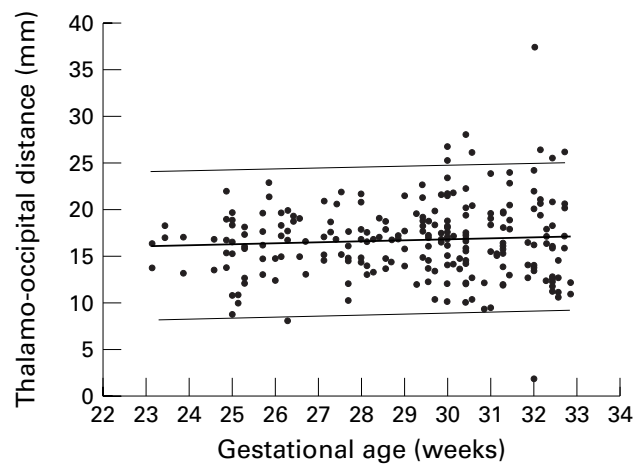

Figure 7 Thalamo-occipital distance (TOD) by gestational age $(G A)$ with fitted line plot (bold line) and $95 \%$ confidence intervals (thin lines). TOD

$(\mathrm{mm})=13.5+0.109 \times G A$ (weeks) $; R^{2}=0.005$.

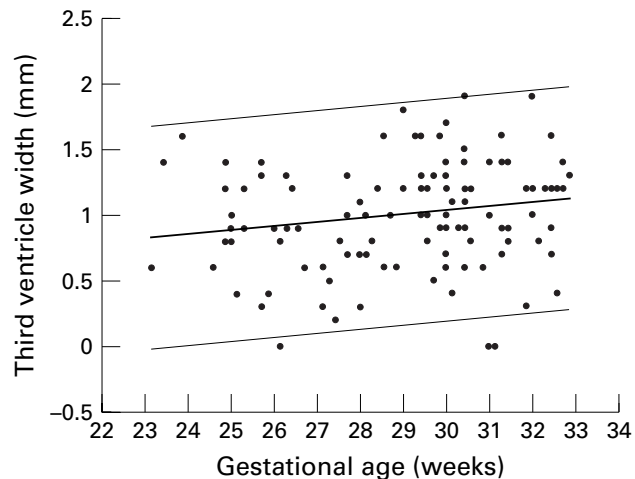

Figure 8 Third ventricle width $\left(3^{\text {rd }} V W\right)$ by gestational age $(G A)$ with fitted line plot (bold line) and 95\% confidence intervals (thin lines). $3^{\text {rd }} V W$

$(\mathrm{mm})=0.064+0.033 \times G A$ (weeks) $; R^{2}=0.038$.

\section{Discussion}

Ultrasound is the preferred method for assessing ventricular size in infants. ${ }^{1}$ Objective measurements are required for the initial diagnosis of ventricular enlargement ${ }^{2}$ and are essential for assessing subsequent changes in ventricular size. Our study provides reference ranges for the linear measurements of all four intracranial ventricles in preterm infants from 23 to 33 weeks' gestational age. This is the first study to provide normal values for measurement of the third and fourth ventricles and the thalamooccipital distance in preterm newborns. These reference ranges can be used to diagnose ventricular enlargement in the preterm infant,

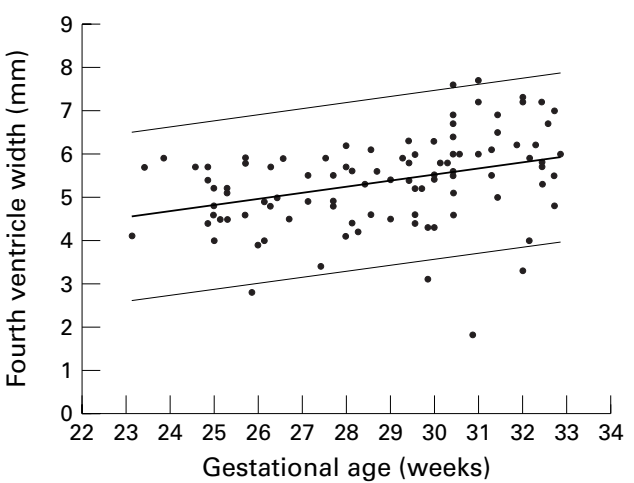

Figure 9 Fourth ventricle width $\left(4^{\text {th }} V W\right)$ by gestational age $(G A)$ with fitted line plot (bold line) and $95 \%$ confidence intervals (thin lines). $4^{\text {th }} V W$

$(\mathrm{mm})=1.33+0.14 \times G A$ (weeks) $; R^{2}=0.124$

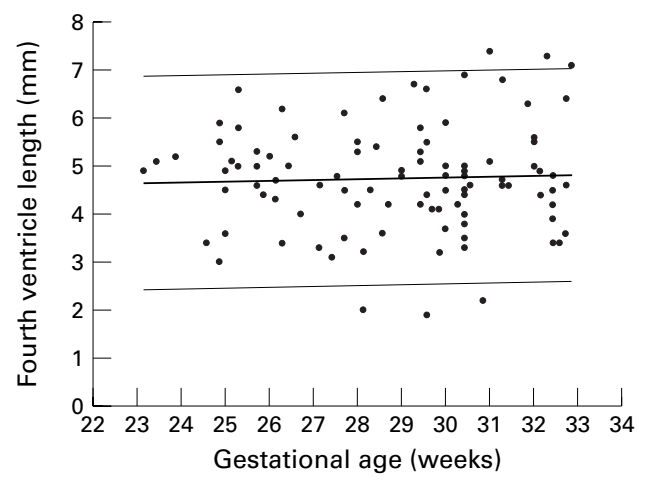

Figure 10 Fourth ventricle length ( $\left.4^{\text {th }} V L\right)$ by gestational age $(G A)$ with fitted line plot (bold line) and 95\% confidence intervals (thin lines). $4^{\text {th }} V L$

$(\mathrm{mm})=4.24+0.017 \times G A$ (weeks) $; R^{2}=0.002$.

and serial measurements are valuable in following the progression of dilatation.

The intraclass correlation coefficient not only takes into account the difference between observations (by a single observer or two different observers), but also the variability between subjects. ${ }^{14}$ All four ventricular measurements are readily reproducible with minimal intra-observer and inter-observer differences. Only the measurement of the fourth ventricle length is moderately unreliable on measurement between observers. The axial resolution of the ultrasound probe is approximately $0.2 \mathrm{~mm}$, which is less than the degree of difference seen within and between observers.

The use of the thalamo-occipital distance to determine enlargement of the occipital horn of the lateral ventricles provides a more subtle marker for ventriculomegaly because the occipital horn is the first to enlarge in posthaemorrhagic ventricular dilatation. ${ }^{16} 17$ The measurement has been used in the fetus in the prenatal diagnosis of hydrocephalus. ${ }^{18}$ Normal values for the measurement of the third and fourth ventricles combined with those for lateral ventricles will also help in differentiating between communicating and noncommunicating ventriculomegaly.

Our results for anterior horn width are consistent with a previous study by Perry et $a l^{13}$ which established an upper limit of $3 \mathrm{~mm}$ in infants from 26 to 42 weeks' gestational age (compared with our upper limit of normal of 
Table 3 Pooled data for intracranial ventricular dimensions from 23 to 32 weeks' gestational age

\begin{tabular}{llll}
\hline Measurement $(\mathrm{mm})$ & Mean & SD & Normal range \\
\hline Anterior horn width $(\mathrm{n}=240)$ & 1.27 & 0.81 & $0-2.9$ \\
Thalamo-occipital distance $(\mathrm{n}=239)$ & 16.7 & 4.0 & $8.7-24.7$ \\
Third ventricle width $(\mathrm{n}=120)$ & 1.07 & 0.74 & $0-2.6$ \\
Fourth ventricle width $(\mathrm{n}=103)$ & 5.37 & 1.03 & $3.3-7.4$ \\
Fourth ventricle length $(\mathrm{n}=102)$ & 4.74 & 1.09 & $2.6-6.9$ \\
\hline
\end{tabular}

Table 4 Difference between two measurements by a single observer for intracranial ventricular measurements

\begin{tabular}{lclll}
\hline Measurement (mm) & $\begin{array}{l}\text { Mean (SD) of average } \\
\text { between measurements }\end{array}$ & $\begin{array}{l}\text { Mean } \\
\text { difference }\end{array}$ & Variability & $\begin{array}{l}\text { Intraclass } \\
\text { correlation } \\
\text { coefficient }\end{array}$ \\
\hline Right AHW & $1.52(0.81)$ & 0.01 & 0.21 & 0.97 \\
Left AHW & $1.70(0.98)$ & 0.01 & 0.25 & 0.97 \\
Right TOD & $16.5(3.9)$ & 0.21 & 0.99 & 0.97 \\
Left TOD & $18.1(4.3)$ & 0.19 & 1.20 & 0.96 \\
Third ventricle width & $1.24(0.32)$ & 0.0 & 0.16 & 0.89 \\
Fourth ventricle width & $5.49(0.91)$ & 0.03 & 0.32 & 0.94 \\
Fourth ventricle width & $5.19(0.89)$ & 0.02 & 0.60 & 0.80 \\
\hline
\end{tabular}

The average between measurements refers to the average of the mean of both repeated measurements.

The mean difference refers to the average difference of the repeated measurements.

Variability refers to the standard deviation of the differences between repeated measurements. AHW, anterior horn width; TOD, thalamo-occipital distance.

$2.9 \mathrm{~mm}$ ). Our thalamo-occipital distance data are similar to fetal data across the same gestational age range. ${ }^{18}$

There is little change in the ventricular dimensions from 23 to 32 weeks' gestational age. The greatest degree of change with gestational age is seen in the fourth ventricle width, with only $12 \%$ of this measurement accounted for by gestational age. This slight increase in fourth ventricle width is probably more related to cerebellar growth, as measured by transverse cerebellar diameter, which shows close correlation with gestational age. ${ }^{19}$ The ventricles are not structures that will grow in the usual sense, because they are merely fluid filled spaces. Growth is accounted for by the laying down of tissue substrate or an increase in cell size or number and this is not applicable to the ventricles. Therefore, it is no surprise that the ventricles do not "grow" as gestational age increases, although the surrounding brain does. Other investigators have shown similar results with regard to measurements relating to the anterior horns. Sauerbrei and colleagues ${ }^{6}$ showed no increase in ventricular index or anterior horn width from 25 to 35 weeks, and Perry and colleagues ${ }^{13}$ also showed no change in anterior horn width from 26 to 42 weeks. In other studies, conducted over large gestational age ranges, changes in dimension of the anterior horns seem to show little increase with age until 30-32 weeks' gestational age. ${ }^{30}$ Saliba et al measured the area of the lateral ventricles and found that they did not increase with gestational age. ${ }^{11}$ There are no other neonatal data with which to compare the thalamooccipital distance, third ventricle width, fourth ventricle width, or fourth ventricle length measurements.

The values need to be interpreted with caution at the lower end of the gestational age spectrum (<25 weeks' gestational age) because of the small number of infants. Recruitment of infants at the margins of viability is difficult, even in a large tertiary centre. However, the measurements from these infants do contribute to the overall regression equation, and the measurements obtained are entirely consistent with the remainder of the data. These results cannot be used to derive conclusions on ventricular size in infants greater than 33 weeks' gestational age. The gestational age range from 23 to 33 weeks was studied because it is this population of babies that has routine cranial ultrasounds soon after birth, because they are most at risk of intraventricular haemorrhage and periventricular leukomalacia. This is also the population that is most at risk for developing post-haemorrhagic ventriculomegaly.

Asymmetry of the lateral ventricles occurs frequently in neonates. ${ }^{21}$ It is a commonly held belief that asymmetry of the lateral ventricles could be the result of the position of the infant's head at the time of the cranial ultrasound, accounted for by the shift of cerebrospinal fluid from the non-dependent to the dependent lateral ventricle. We have shown that head position has no effect on the difference in measurements between the nondependent and dependent lateral ventricles. Similarly, the sex of the infant has little influence on ventricular size.

\section{Conclusions}

References ranges for the measurement of the intracranial ventricles in preterm infants from 23 to 33 weeks' gestational age are provided. These measurements can be used in the diagnosis and assessment of ventricular enlargement in preterm infants less than 33 weeks' gestational age. All measurements have good intra-observer and inter-observer reliability. Head position at the time of the scan does not influence the asymmetry of the lateral ventricular measurements. The infant's sex does not significantly influence ventricular size.

1 Rennie JM. Enlarged cerebral ventricles. In: Neonatal cerebral ultrasound. Cambridge: Cambridge University Press, 1997:155-69.

2 Shackleford GD. Neurosonography of hydrocephalus in infants. Neuroradiology 1986;28:452-62.

3 Levene MI. Measurement of the growth of the lateral ventricles in preterm infants with real time ultrasound. Arch Dis Child 1981;56:900-4.

4 Allan WC, Holt PJ, Sawyer LR, Tito AM, Meade SK. Ventricular dilatation after neonatal periventricularintraventricular hemorrhage. Natural history and therapeutic implications. Am f Dis Child 1982;136:58993.

5 London DA, Carroll BA, Enzmann DR. Sonography of ventricular size and germinal matrix hemorrhage in premature infants. AFNR Am f Neuroradiol 1980;1:295-300.

6 Sauerbrei EE, Digney M, Harrison PB, Cooperberg PL. Ultrasonic evaluation of neonatal intra-cranial hemorrhage and its complications. Radiology 1981;139:677-85.

7 Skolnick ML, Rosenbaum AE, Matzuk T, Guthkelch AN, Skolnick ML, Rosenbaum AE, Matzuk T, Guthkelch AN,
Heinz ER. Detection of dilated cerebral ventricles in Heinz ER. Detection of dilated cerebral ventricles in
infants: a correlative study between ultrasound and infants: a correlative study between ultrasound
computed tomography. Radiology 1979;131:447-51.

8 Levene MI, Starte DR. A longitudinal study of posthaemorrhagic ventricular dilatation in the newborn. Arch Dis Child 1981;56:905-10.

9 Helmke K, Winkler P. Sonographisch ermittelte normwerte des intrakraniellen ventrikelsystemes im ersten lebensjahr. Monatsschr Kinderheilkd 1987;135:148-52.

10 Poland RL, Slovis TL, Shankaran S. Normal values for ventricular size as determined by real time sonographic techniques. Pediatr Radiol 1985;15:12-14.

11 Saliba E, Bertrand P, Gold F, Vaillant MC, Laugier J. Area of lateral ventricles measured on cranial ultrasonography in preterm infants: reference range. Arch Dis Child 1990;65:1029-32. 
12 Papile L, Burstein J, Burstein R, Koffler H. Incidence and evolution of subependymal and intraventricular hemor-
rhage: a study of infants with birth weights less than rhage: a study of infants with birth

13 Perry RNW, Bowman ED, Roy RND, de Crespigny LC Ventricular size in newborn infants. 7 Ultrasound $\mathrm{Med}$ 1985;4:475-7.

14 Jamart J. Assessing observer variability. BMF 1992;305:31312.

15 Brennan P, Silman A. Statistical methods for assessing observer variability in clinical measures. $B M \mathcal{F}$ 1992;304:1491-4.

16 Brann BS, Qualls C, Wells L, Papile L. Asymmetric growth of the lateral cerebral ventricle in infants with posthemorrhagic ventricular dilatation. F Pediatr 1991;118:108-12. 17 Silverboard G, Horder MH, Ahmann PA, Lazzara A,
Schwartz JF. Reliability of ultrasound diagnosis of intracerebral hemorrhage and posthemorrhagic hydrocephalus: comparison with computed tomography Pediatrics 1980;66:507-14.

18 Monteagudo A, Haratz-Rubinstein N, Timor-Tritsch IE. Biometry of the fetal brain. In: Timor-Tritsch IE, Monteagudo A, Cohen HL, eds. Ultrasonography of the prenatal and neonatal brain. Connecticut: Appleton and Lange, 1996:89-146.

9 Swaminathan M, Davies MW, Davis PG, Betheras FR. Transverse cerebellar diameter on cranial ultrasound scan in preterm neonates in an Australian population. $\mathcal{F}$ Paediatr Child Health 1999;35:346-9.

20 Liao M, Chaou W, Tsao L, Nishida H, Sakanoue M. Ultrasound measurement of the ventricular size in newborn infants. Brain Dev 1986;8:262-8.

21 Shen E, Huang F. Sonographic finding of ventricular asymmetry in neonatal brain. Arch Dis Child 1989;64:730-44. 